\title{
Fault Detection and Isolation Based on Neural Networks Case Study: Steam Turbine
}

\author{
Djamel Benazzouz, Samir Benammar, Smail Adjerid \\ Solid Mechanic and Systems Laboratory (LMSS), M'Hamed Bougara University, Boumerdès, Algeria \\ E-mail: dbenazzouz@yahoo.fr \\ Received February 9, 2011; revised March 20, 2011; accepted April 8, 2011
}

\begin{abstract}
The real-time fault diagnosis system is very important for steam turbine generator set due serious fault results in a reduced amount of electricity supply in power plant. A novel real-time fault diagnosis system is proposed by using Levenberg-Marquardt algorithm related to tuning parameters of Artificial Neural Network (ANN). The model of novel fault diagnosis system by using ANN are built and analyzed. Cases of the diagnosis are simulated. The results show that the real-time fault diagnosis system is of high accuracy and quick convergence. It is also found that this model is feasible in real-time fault diagnosis. The steam turbine is used as a power generator by SONELGAZ, an Algerian company located at Cap Djinet town in Boumerdes district. We used this turbine as our main target for the purpose of this analysis. After deep investigation, while keeping our focus on the most sensitive parts within the turbine, the weakest and the strongest points of the system were identified. Those are the points mostly adequate for failure simulations and at which the designed system will be better positioned for irregularities detection during the production process.
\end{abstract}

Keywords: Failure, Diagnosis, Artificial Neural Networks, Isolation, Steam Turbine

\section{Introduction}

The first role of the industrial diagnosis is to increase the availability of the industrial installations to reduce the direct and indirect maintenance costs of the production equipments. The direct costs of this maintenance are mainly those related to the various spare parts. On the other hand, the indirect costs are essentially due to the off line production [1,2]. The increase repair time influences negatively on the indirect maintenance costs.

The objective of this paper is to minimize this waiting time for detecting the failure in the industrial installations. The proposed model will supervise the system, detect and localize any faulty in real time. An important characteristic of the proposed model is that it has the possibility of detecting and locating several failing points at the same time. For example: an increase in the vibration level in the four landings of the turbine. The data vectors for the training in the Artificial Neural Network (ANN) model are intervals limited by two values, minimum and maximum. The used symbol " 1 " represents a normal functioning and the symbol " $\mathbf{- 1}$ " represents a failure situation. The training algorithm used for the network is the Levenberg-Marquardt algorithm, the choice of this algorithm is that it gives a fast training of the ANN compared to the other algorithms of decent of gradient $[3,4]$. The programming was completely developed under MATLAB 7.5.

\section{Steam Turbine Presentation}

The study case concerns a steam turbine of an Algerian electrical production thermal plant SONELGAZ located at Cap-Djinet, Boumerdes. The turbine transforms the thermal energy contained in the vapor coming from the boiler into a rotation movement of the tree. Mechanical work obtained is used to actuate the alternator. It is composed of three bodies, HP body (High Pressure), MP body (Average Pressure) and BP body (Low Pressure). It has a power and a nominal number of revolutions of 176 MW and $3000 \mathrm{rpm}$ respectively. The line of tree rests on four landings, each one of these landings thus carries two relative vibration sensors, it is the total of eight sensors on all the line of tree, but for model simplification we consider only four sensors. The maximum value of relative vibrations that can be supported by the system is 120 $\mu \mathrm{m}$. Figure 1 represents the supervision and placement sensors site in the landings turbine. 


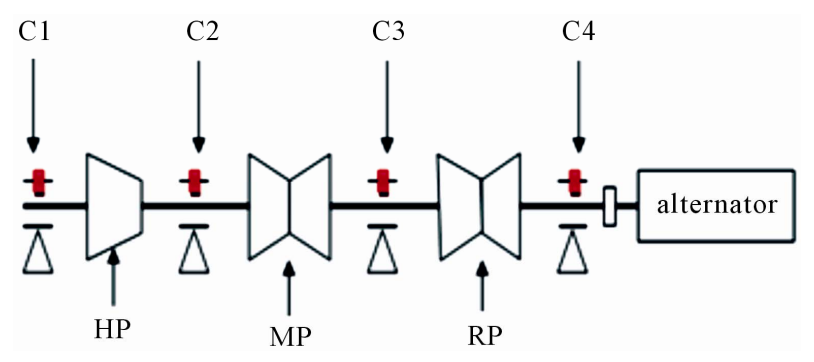

Figure 1. Sensor vibrations site in the turbine landings C1, C2, C3 and C4 are respectively the supervision sensors of the tree relative vibrations compared to landings $1,2,3$ and 4 .

\section{Calculation of the Optimal Neural Network Architecture}

There exist many applications of ANN in industry particularly in data analysis, model and command identification [4,5]. Among various types of ANN, the most used is the multi-layer perceptron (MLP) which is retained in our application as a powerful tool. A network MLP is generally composed by one neural entry layer, one or more hidden in the intermediate layers and one output layer. Figure 2 shows the proposed architecture model where the input vector is $X=\left[x_{1}, x_{2}, \cdots x_{M}\right]^{T}$, with $M=951$, the hidden layer neurons varies from $\mathrm{j}$ to $N$ with $N=15$ and then the output vector

$Y=\left[y_{1}, y_{2}, y_{3}, y_{4}\right]$. The general laws for the calculation of the hidden layer neurons and the output layer are respectively:

$$
\begin{gathered}
u_{f}=f_{1}\left(\sum_{i=1}^{M} w_{i, j}^{1} \cdot x_{i}+b_{j}^{1}\right) \\
y_{k}=f_{2}\left(\sum_{l=1}^{N} w_{l, k}^{2} \cdot u_{l}+b_{k}^{2}\right)
\end{gathered}
$$

$f_{1}, f_{2}$ are respectively the sigmoid activation (3) and linear functions (4).

$$
\begin{gathered}
f_{1}(v)=\frac{2}{1+e^{-2 v}}-1 \\
f_{2}(v)=v
\end{gathered}
$$

The network training MLP implies to find connection weights values $w_{i, j}^{1}$ and $w_{l, k}^{2}$, which reduce to the minimum the average error function (MSE) between the measured value and the theoretical (desired) value corresponding in the training step. The network training by the Levenberg-Marquardt algorithm is constructed directly using Matlab neural network toolbox [6,7].

The optimal architecture, after several trials, was found by this configuration "951-12-04" which gives the smallest error of $1.90756 \mathrm{e}^{-005}$, after 111 iterations, during $113.86 \mathrm{sec}$.

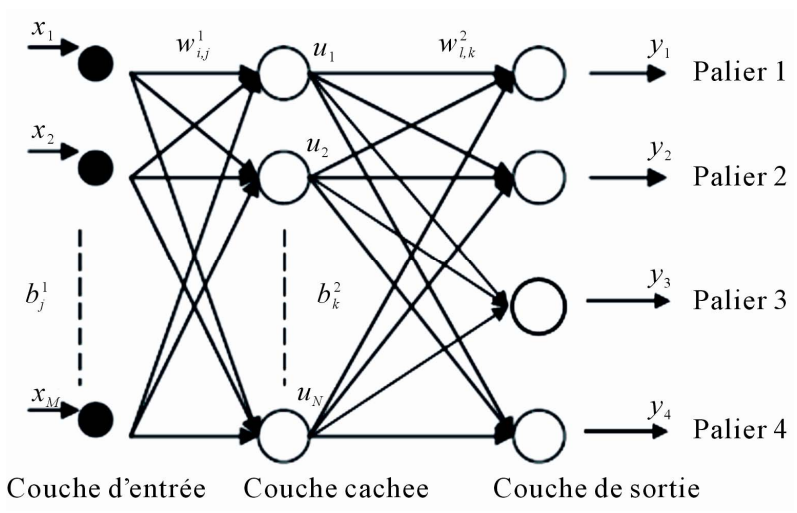

Figure 2. Proposed network structure.

$$
M S E=\frac{1}{n} \sum_{k=1}^{n}\left(d-y_{k}^{2}\right)^{2}
$$

$n$ : number of examples.

1) Flowchart for the Calculation of Optimal Architecture

$W, b$ : are the weight matrix and the bias vector (the initialization of the weight and bias values is taken randomly between $[-1,+1])$. The following symbols are used,

"it": iterations number,

Ei:(internal excitation) desired error,

$\mu$ : training rate (between 0 and 1 ),

$f_{1}$ : activation function of the hidden layer,

$f_{2}$ : activation function of the output layer,

Aap: training algorithm,

NNS: neurons number in the output layer,

$p$ : the step,

$\mathrm{Ni}$ : desired number,

$R$ : correlation coefficient,

$R G$ : generalized network coefficient,

$M S E$ : mean square error,

Tap: training time,

Ti: training desired time.

To obtain optimal architecture we must firstly vary the neurons number of the hidden layer $(N)$ to 1 until reaching the desired number (Ni) to satisfy condition MSE $\leq$ Ei.

(Quadratic average error $\leq$ desired error), if there is no value of $N$ which satisfies this condition, we must return to the network parameters to fix another initials values. In the second step we observe the behavior of the network and identify all the correlation factors which should be at " 1 ". If it is not we should increase the desired error (Ei). The last step is the generalization phase. It consists of calculating the correlation coefficient of the generalized network RG which must be close to "1" as shown by the developed flowchart in Figure 3. 


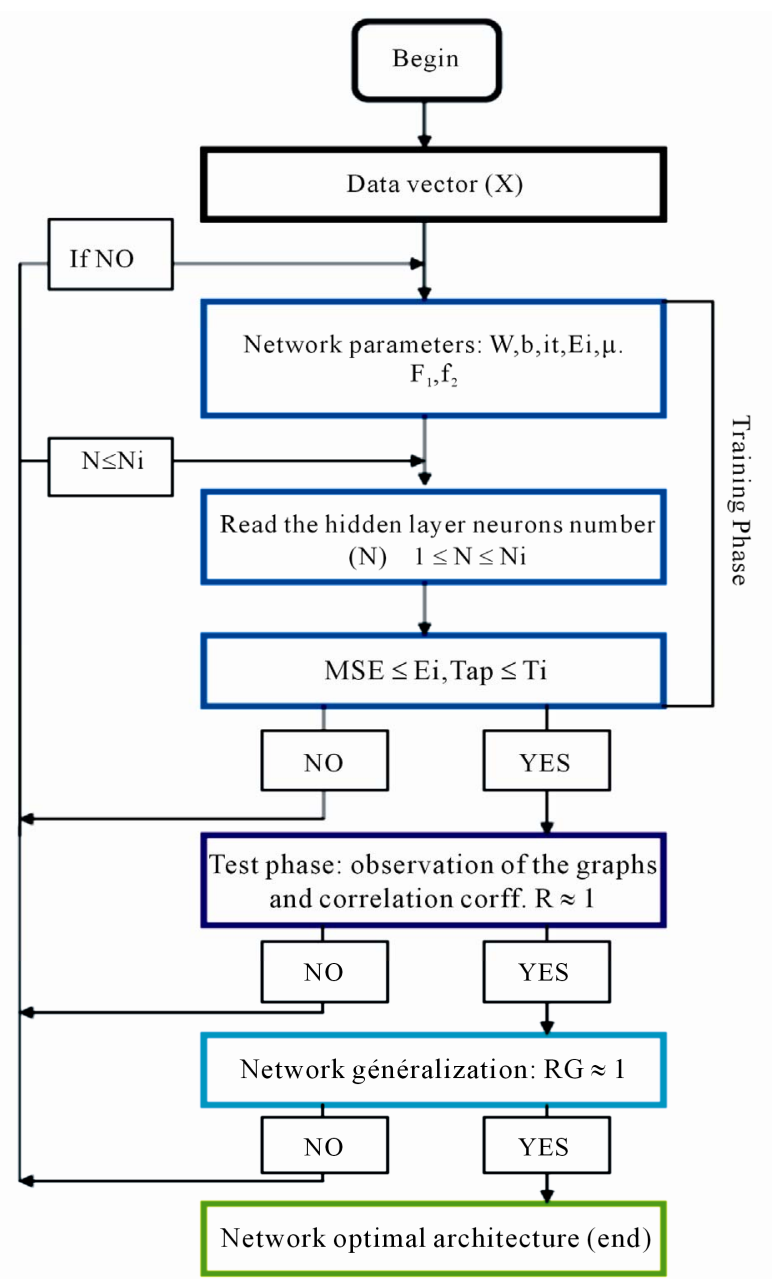

Figure 3. General flowchart for the choice of an optimal architecture.

\section{2) Network Training}

The training graph (Figure 4) converges towards an error of the order of $10^{-4}$ after 111 iterations during $113.86 \mathrm{sec}$.

\section{3) Output Network Simulation}

We observe in Figure 5, the network graphs in continuous line, superpose with the desired functions graphs in dashed line. This allows us to say that it is an acceptable architecture since the error is of the order of $10^{-4}$.

Vrp1: relative vibrations for landing 1,

Vrp2: relative vibrations for landing 2,

Vrp3: relative vibrations for landing 3,

Vrp4: relative vibrations for landing 4,

Svp1: desired output of the relative vibrations of landing 1 ,

Svp2: desired output of the relative vibrations of landing 2,

Svp3: desired output of the relative vibrations of landing 3,

Svp4: desired output of the relative vibrations of landing 4 .

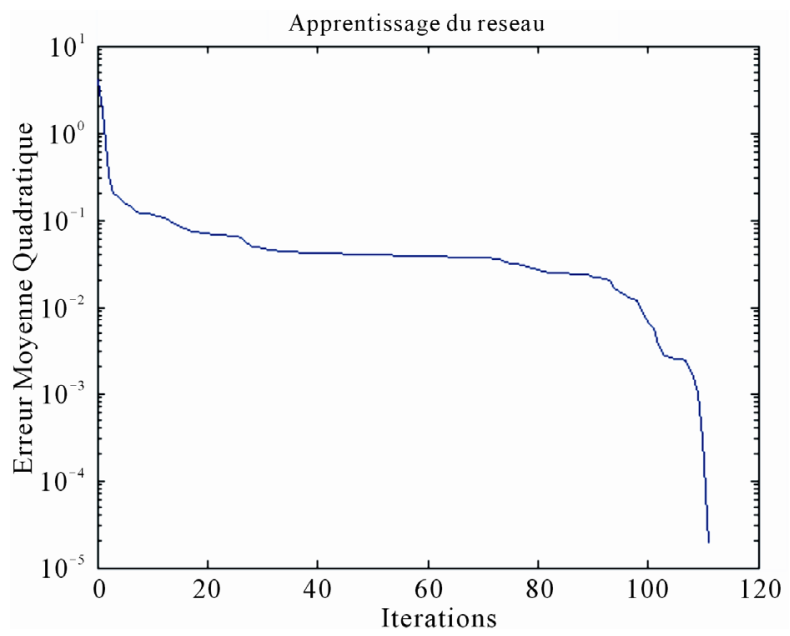

Figure 4. Training of the founded neural network.

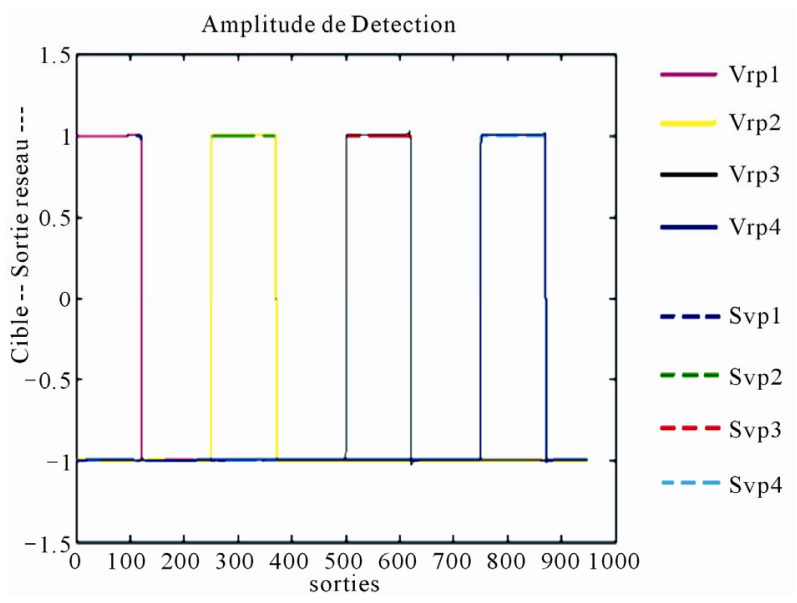

Figure 5. Output network simulation.

4) Fault Detection and Localization (Test of the Network)

To be sure that the proposed network will detect any faulty we will try to inject a known faulty and see the behavior of the network. This is presented in the following examples.

Example 1: We consider 3 correct values and 1 incorrect value, for landing 1 is $160 \mu \mathrm{m}$, for landing 2 is 110 $\mu \mathrm{m}$, for landing 3 is $100 \mu \mathrm{m}$ and $90 \mu \mathrm{m}$ for landing 4 .

Figure 6 shows the unacceptable value test, where the curve does not superpose the learned one.

Example 2: In this example we will generalize our test to give random intervals which do not belong to the acceptable interval. Instead of considering value, now we consider a set of values. We test by set values $[120 \mu \mathrm{m}$ $239 \mu \mathrm{m}]$ for Vrp1, [300 $\mu \mathrm{m}-419 \mu \mathrm{m}]$ for $\operatorname{Vrp} 2$, [120 $\mu \mathrm{m}$ - $239 \mu \mathrm{m}$ ] for Vrp3 and by [400 $\mu \mathrm{m}-519 \mu \mathrm{m}$ ] for Vrp4. The network output detects four alarms in the four points of measurement as shown in Figure 7 due to an unacceptable vibrations values given to the network. 


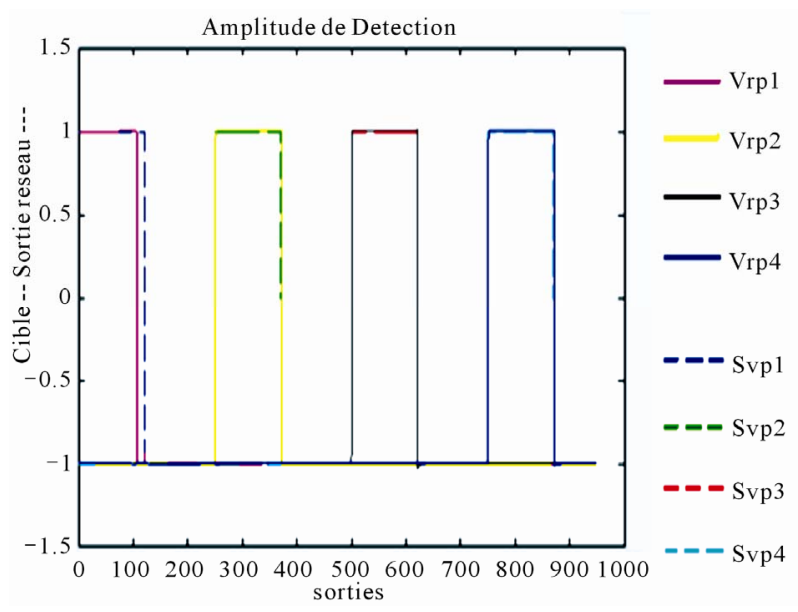

Figure 6. Unacceptable value test.

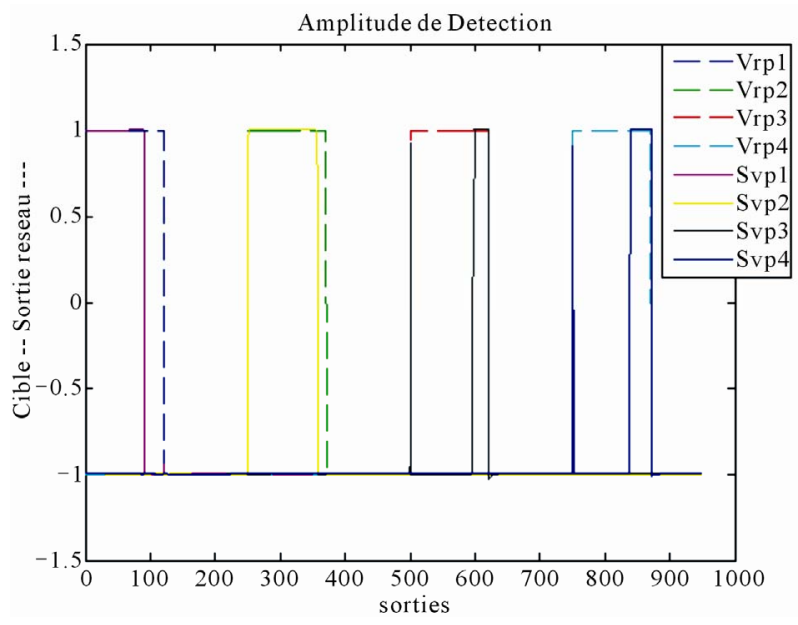

Figure 7. Unacceptable vibrations under a set of values test.

\section{5) Performance Evaluation}

In Figure 8 we show the correlation coefficient " $R$ " which tends towards " 1 ", this fulfill the condition correlation requirement as mentioned before and confirm that the output values are compared to the desired output values in the network. This is represented in Figure 8 by superposing the continuous line which is the best linear adjustment fit between the targets and the network outputs (dashed line).

\section{Conclusions}

In this investigation, we have proposed MLP architecture for detecting and locating any faulty in the monitored system using the Levenberg-Marquardt algorithm. That is due to its speed and training availability.

The suggested network system will help the maintenance team to better localize and automatically identify the sources of failures. This way, all they will have to do is to fix the defective parts, which will improve the net-

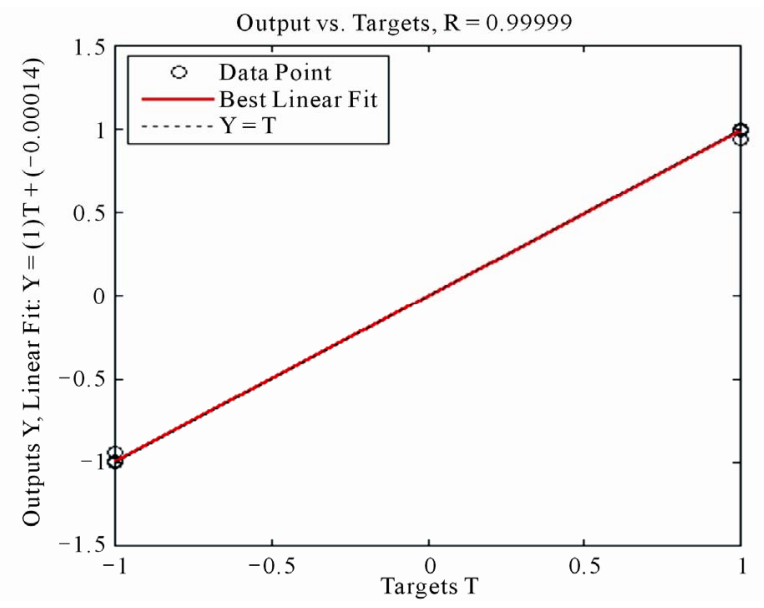

Figure 8. Performance of the proposed network.

work maintenance operations by reducing both the time and cost of troubleshooting.

\section{References}

[1] M. Basseville and M.-O. Cordier, "Surveillance et DiagNostic de Systèmes Dynamiques: Approche Complémentaire du Traitement de Signal et de l'Intelligence ArtifiCielle," Rapport INRIA, Thèmes 3-4, No. 2861, 1996, pp. $1-40$.

[2] N. Palluat, D. Racoceanu and N. Zerhouni, "Utilisation des Réseaux de Neurones Temporels pour le Pronostic et la Surveillance Dynamique," Etude Comparative de Trois Réseaux de Neurones Récurrents, RSTI-RIA, Vol. 19, No. 6, 2005, pp. 911-948.

[3] M. Bouamar and M. Ladjal, "Système Multicapteur Utilisant les Réseaux de Neurones Artificiels pour la Surveillance des Eaux Potables," 4th International Conference: Sciences of Electronic, Technologies of Information and Telecommunications, LASS, Laboratoire d'Analyse des Signaux et Systèmes, Université de M'sila, Algérie, 25-29 March 2007.

[4] M. I. A. Lourakis, "A Brief Description of the Levenberg-Marquardt Algorithm," Implemented Institute of Computer Science Foundation for Research and Technology-Hellas (FORTH) Vassilika Vouton, Heraklion, 2005.

[5] J. Karim, "Surveillance, Diagnostic et Pronostic en Temps Réel de Systèmes Hybrides: Application à des Bancs d'Essais CERTIA," LAAS-CNRS Groupe DISCO 7, Toulouse, 2007.

[6] T. Alani, "Réseaux de Neurones Tutorial en Matlab," Département Informatique ESIEE-Paris, Paris, 2008.

[7] M. S. Patil, Jose Mathew and P. K. R. Kumar, "Bearing Signature Analysis as a Medium For Fault Detection: A Review," Journal of Tribology, Vol. 130, No. 1, 2008, pp. 1-7. doi:10.1115/1.2805445 